\title{
Modelagem e validação de um sistema multiplataforma para Gerenciamento de Energia Elétrica - SISGEE
}

\author{
*1I. S. de Carvalho; *2'U. C. Paixão Jr.; *A. C. D. B. de Souza; *J. R. da S. Muniz; *F. P. Monteiro; *M. E. de L. Tostes \\ *Centro de Excelência em Eficiência Energética da Amazônia, Universidade Federal do Pará, Belém - PA \\ ${ }^{1}$ izidiocarvalho88@gmail.com \\ 2 upaixaojr.eng@gmail.com
}

\begin{abstract}
Several sectors are looking for ways to obtain surveys and studies related to the consumption and quality of electricity from the electric system, so, given this motivation, a power management software, called the Electric Power Management System - SISGEE, was developed. The software is able to assist in the tasks of analysis of efficiency, quality and consumption of electricity, through graphs, tables, state of operation and geolocation of the various meters installed on the campus of the Federal University of Pará (UFPA), which perform the full-time data collection on an autonomous basis. The present work focuses on the modeling of the SISGEE WEB module, presenting the paradigms of responsiveness, material design and MVC (model, visualization and control). Thus, the article aims to present the stages of software development and implementation, as well as the validation of the main input data (voltage and current) that serve as the basis for calculating the other quantities of software analysis.

Resumo: Diversos setores buscam formas para obter levantamentos e estudos relacionados ao consumo e à qualidade da energia elétrica proveniente do sistema elétrico, então, dado a esta motivação, foi desenvolvido um software de gestão de energia, chamado de Sistema de Gestão de Energia Elétrica - SISGEE. O software é capaz de auxiliar nas tarefas de análise de eficiência, qualidade e consumo de energia elétrica, por meio de gráficos, tabelas, estado de funcionamento e geolocalização dos diversos medidores instalados no campus da Universidade Federal do Pará (UFPA), que realizam a coleta de dados em tempo integral e de forma autônoma. O presente trabalho toma como enfoque principal a modelagem do módulo WEB do SISGEE, apresentando os paradigmas da responsividade, do material design e do MVC (modelo, visualização e controle). Sendo assim, o artigo tem por objetivo apresentar as etapas de desenvolvimento e implementação do software, assim como a validação dos principais dados de entrada (tensão e corrente) que servem como base para calcular as demais grandezas de análise do software.
\end{abstract}

Keywords: Multiplataform; Web System; Energy management; Quality of Electric Power.

Palavras-chaves: Multiplataforma; Sistema Web; Gestão de Energia; Qualidade de Energia Elétrica.

\section{INTRODUÇÃO}

Com o avanço da tecnologia, houve o aumento no número de dispositivos computacionais capazes de realizar tarefas que, em um passado recente, somente os computadores poderiam exercer, como a execução de softwares mais complexos, com alta demanda de desempenho de hardware, e a conectividade com a Internet. Tal avanço proporcionou que diversos fabricantes introduzissem os mais diversos dispositivos no mercado mundial [1], sejam estes mainframes, desktops pessoais ou dispositivos móveis.

Acompanhando o avanço tecnológico, os mais diversos setores sociais buscam meios de usar os aparatos tecnológicos em proveito de melhores resultados em seu campo de ação. Dito isto, há alguns anos a crise energética tem se tornado assunto frequente nos principais debates mundiais sobre a crise energética, o desenvolvimento do país e a sustentabilidade. No que tange ao cenário nacional, nos últimos anos, tanto a geração quanto o consumo energético possuem aumento de visibilidade em virtude das recorrentes crises no setor por conta da utilização de fontes de energia não renováveis; a intermitência do período chuvoso e o impacto ambiental da geração de energia elétrica (hídrica); a crescente demanda de energia; o alto custo de transmissão; e a inserção da geração distribuída com outras fontes renováveis, como a solar, eólica e biomassa. Ainda neste cenário, o setor elétrico está propício ao agravante do desperdício de energia, devido à má utilização pelos usuários finais; as perdas técnicas, ocasionadas pelas longas linhas de transmissão e equipamentos eletrônicos; e as perdas não técnicas ocasionadas, principalmente, por furtos de energia [2].

Dentro desse contexto, a equipe do Centro de Excelência em Eficiência Energética da Amazônia (CEAMAZON) criou o software Sistema de Gestão de Energia Elétrica (SISGEE), modelado para atender às diversas demandas de conectividade em diversos sistemas operacionais, tais como acessos via desktop, tablets e smartphones. O SISGEE é capaz de auxiliar as equipes de manutenção, os gestores e os usuários finais a monitorar o consumo e a qualidade da energia elétrica, além de gerar indicadores que refletem o grau de eficiência, economia e qualidade da energia das instalações consumidoras de forma didática e rápida.

O objetivo deste trabalho é apresentar as ferramentas utilizadas para criação do software, com foco no módulo web do sistema de gestão de energia elétrica, além da validação de grandezas elétricas (tensão e corrente) monitoradas por meio do mesmo. Os dados empregados para validação foram medidos no ponto 
de acoplamento comum (PAC) entre a universidade e a concessionária local.

\section{SISTEMA DE GESTÃO DE ENERGIA ELÉTRICA}

A estrutura de funcionamento do SISGEE está dividida em três agrupamentos, sendo o medidor (sistema de medição comercial), usuários em diversas plataformas (desktop, notebook, tablet, celular, etc.) e servidor (local de armazenamento do software desenvolvido). A Fig. 1 ilustra os meios físicos mencionados.

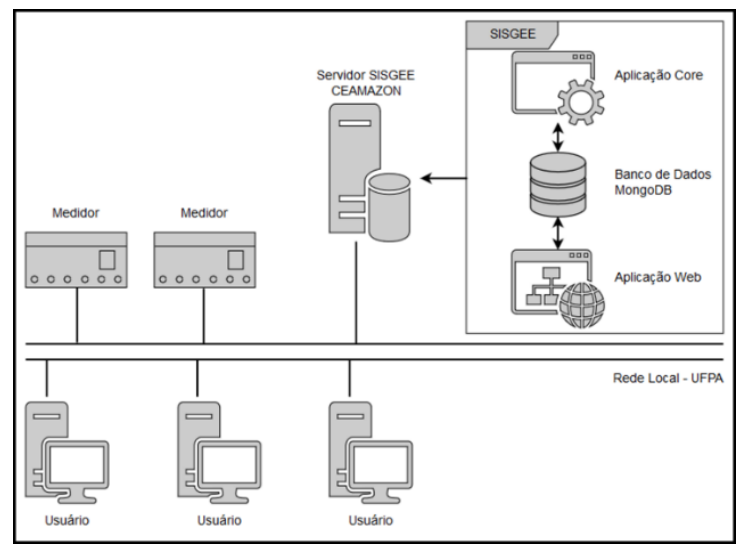

Fig. 1 - Arquitetura física do SISGEE.

Para medição foram utilizados medidores da fabricante Embrasul, modelo TR4000, capaz de medir a tensão e a corrente no intervalo de dez minutos, além de fornecer o cálculo de grandezas como consumo, potência (ativa, reativa e aparente), distorção harmônica total (tensão e corrente) e fator de potência.

A facilidade de acesso ao software e às informações são os grandes destaques do SISGEE, haja vista que o mesmo utiliza as mais modernas técnicas de visualização para garantir total usabilidade do sistema e facilidade na extração de informações. Com isto, enxergar o máximo de unidades em tempo real minimiza o trabalho de técnicos e operadores na manutenção do campus.

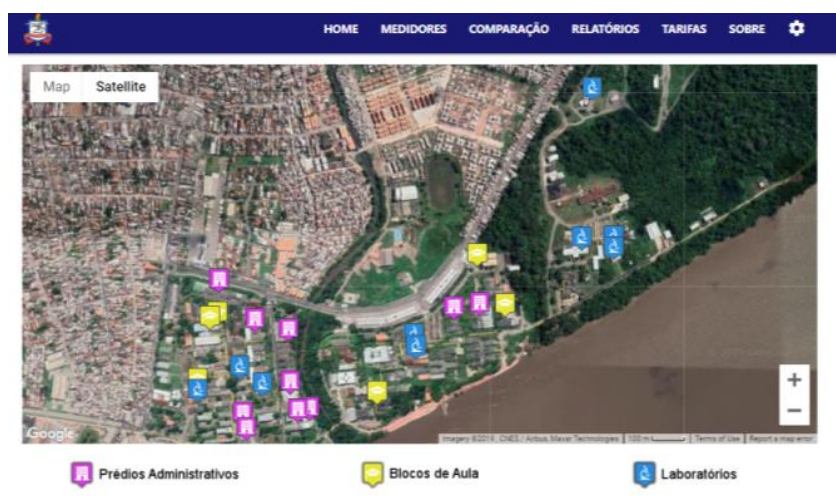

Fig. 2 - Página inicial do software SISGEE.

Na Fig. 2, tem-se a tela inicial do SIEGEE, que apresenta as abas com as funções do software. Como tela principal, a aba medidores apresenta as grandezas e possibilita a escolha dos períodos de análise; as demais abas são mais intuitivas e permitem a comparação e a emissão de relatórios, assim como a análise tarifária dos medidores; por último, a aba sobre descreve equações e breves explicações conceituais sobre o software.

\section{MODELAGEM DO SOFTWARE SISGEE}

O projeto SISGEE foi elaborado em três camadas de desenvolvimento na finalidade de obter ganho de produtividade, compreendendo o desenvolvimento em paralelo nas camadas e o ganho em escalabilidade do sistema através da independência entre cada camada de desenvolvimento.

As ferramentas adotadas na construção do SISGEE, também foram empregados em diversas soluções, utilizando os mesmos moldes do contexto da criação de softwares e estudos, sendo o Python foi utilizado pelos softwares Hidralerta [3] e UbibusRoute [4]. De modo semelhante ao Python, o MVC e o Modbus também foram empregados para desenvolver um sistema de monitoração de dados de uma refinaria de petróleo [5].

Assim como a criação do Sistema inteligente de recolha, armazenamento e visualização de informação proveniente do Twitter, teve como base de dados o MongoDB, por conta do grande volume de informações a serem armazenadas, lidas e processadas [6].

\subsection{Sistema Core}

É responsável por fazer a aquisição dos dados via redes de computadores, o controle de qualidade destes, e por desempenhar o papel de comunicação entre o software desenvolvido e os medidores. A comunicação para a requisição de dados ocorre por meio do protocolo Modbus na camada de aplicação, camada 7 do modelo OSI (Open System Interconnection) [7] e Python como linguagem de programação.

\subsubsection{Protocolo Modbus}

O Modbus é um protocolo de redes atuante na sétima camada do modelo OSI, na qual sintetiza a comunicação entre máquinas heterogêneas através da definição de estruturas genéricas para a construção de metodologias para troca de informações que independem da tecnologia, sem que haja a necessidade de modificação no software ou no hardware dos equipamentos envolvidos [8].

O protocolo implementa a arquitetura cliente/servidor com 4 estágios para o estabelecimento de comunicação e troca de informações. Com isto, ao dispositivo incumbido de solicitar a informação é dado o nome de cliente e o dispositivo que irá transmitir a resposta da solicitação é tratado como servidor.

Desta forma a Fig. 3 exemplifica o ciclo de troca de informações entre cliente e o servidor, em que na primeira etapa, o cliente inicia uma requisição, envia uma mensagem ao servidor contendo o código da função e as informações da requisição; em seguida, o servidor recebe essa requisição e executa o comando; na terceira etapa é enviada uma resposta à 
solicitação juntamente com o código da função; e, por fim, o cliente recebe a resposta [9].

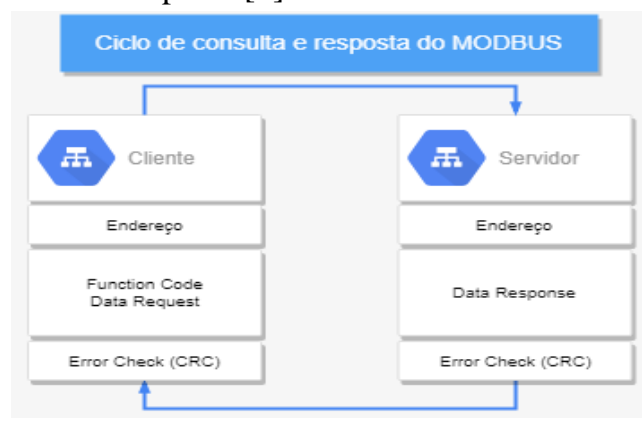

Fig. 3 - Ciclo de consulta do Modbus.

\subsubsection{Linguagem Python}

A linguagem de programação escolhida para o Core foi o Python, dentre os motivos podemos destacar o fato do mesmo ser um sistema com código aberto, implementando linguagem de alto nível e multiplataforma, uma vez que se pode instalar o compilador em diversos sistemas operacionais.

O Python implementa os conceitos de orientação a objetos e roda em formato de script, por esse motivo é muito utilizado para preparar rotinas recorrentes e automatizadas. Tendo em vista sua estrutura, a linguagem fornece a robustez e a facilidade necessária para a criação de scripts com alto desempenho computacional, de forma simples e rápida. Por este motivo o Python é muito difundido nas áreas de mineração de dados, Big Data, reconhecimento de padrões, inteligência computacional e aprendizado de máquina supervisionada e não supervisionada [10].

\subsubsection{Aquisição e tratamento de dados}

O Python é utilizado para implementar o acesso ao medidor por meio do protocolo Modbus, requisitando as medidas instantâneas. Após a obtenção ocorre a integralização das medidas e a conversão das séries de medidas instantâneas integralizadas em um período de 10 minutos utilizando o critério de Chauvenet para a remoção de medidas discrepantes em relação à média, conforme pode ser visto em [11]. Tal ação busca atender o módulo 8 dos Procedimentos de Distribuição (PRODIST) da Agência Nacional de Energia Elétrica (ANEEL) que determina 1008 medições no intervalo de uma semana para análise da qualidade de energia elétrica [12].

Além do Critério de Chauvenet, a cada intervalo de 1 hora, são realizados testes periódicos relacionados a permanência das informações contidas nos medidores, visando analisar informações como data e hora do medidor e o número de série, para garantir que a leitura dos dados seja feita corretamente.

\subsection{Módulo de Database}

Este módulo é responsável por manter a persistência da informação, pois, para ter acesso a qualquer conteúdo como fotos, imagens, textos ou vídeos, estes precisam estar acessíveis fisicamente. Entretanto, à medida em que a quantidade de acessos e arquivos aumenta, a complexidade de armazenamento e acesso também aumenta.
Conforme descrito por [13] e utilizado neste trabalho, a tecnologia de bancos de dados foi desenvolvida para suprir a demanda referente à organização física e lógica dos dados, em resposta aos obsoletos sistemas de arquivos usados para consulta de dados, de forma a garantir a permanência da informação armazenada.

Pesquisas têm sido realizada para melhorar a capacidade de armazenamento e o tempo de acesso as informações da rede, com isto, surgiram os primeiros bancos de dados em SQL. Atualmente os bancos de dados NoSQL são uma forma de armazenamento, visando a melhoria da performance e da escalabilidade em relação ao grande volume de dados gerados diariamente, como exemplo as redes sociais e as redes de sensores, dando origem ao Big Data.

Diferente do sistema gerenciador de banco de dados (SGBD) em que os dados são armazenados de forma lógica em tabelas e a notação do SQL como linguagem, o MongoDB é um Banco de Dados NoSQL (do inglês, not only $S Q L$ ) que armazena a informação lógica em forma de documentos, utilizando JSON. Desta forma, permite que o acesso à informação aconteça de forma dinâmica e em maior volume.

\subsection{Software Web}

Ao longo do tempo, os computadores pessoais foram os responsáveis por grande parte dos acessos e conexões na internet ou intranet, de modo que os dispositivos dispunham de configurações semelhantes e eram constituídos basicamente por um teclado, mouse e uma tela de tamanho convencional. Atualmente o uso dos equipamentos smarts estão aumentando progressivamente a nova era das conectividades, sendo estas encabeçadas pelos smartphones e conceitos como internet of things (IoT).

As características construtivas do sistema, como a modelagem e manutenção, também sofreram evoluções, pois era comum o desenvolvimento de um sistema estar na mesma camada das ações dos usuários, das regras de negócios e do acesso aos dados. Contudo, esta abordagem dificultava o desenvolvimento e manutenção do código, logo, surgiu a necessidade de padronização do processo de criação de sistemas [14].

A popularização dos computadores e a produção dos sistemas voltados a atender as demandas do mercado, fez com que a interface gráfica se torne tão importante quanto as demais, pois é a camada do sistema que está mais visível e próxima do usuário, logo, esta deve ser considerada como um aspecto pertinente na qualidade de um software[15].

Responsável por exibir as informações obtidas e processadas, o software web do SISGEE atua como interface de comunicação com o usuário, sendo a camada interativa, visual e mais externa do mesmo. Para tal, o software web é dividido em três princípios: (1) estrutural, sistema MVC atuante na arquitetura interna do software; (2) design, na forma de linguagem visual a fim de obter um design moderno e agradável, (3) acessibilidade, por meio de múltiplas plataformas através do uso da responsividade. 


\subsubsection{Model, View e Controller - MVC}

Para este projeto, a estrutura MVC foi adotada por compreender aspectos inerentes ao processo de desenvolvimento de projetos de softwares, tais como a organização interna do fluxo de informação lógica no sistema e o fornecimento de um conjunto geral de métodos genéricos reutilizáveis durante a execução do software.

A estrutura MVC é composta por três tipos de objetos que constituem as camadas da arquitetura, sendo o modelo (Model), onde as requisições da camada de controle (Controller) são processadas. O modelo dita as regras do negócio, possui a conectividade com o banco de dados e os métodos de propósitos gerais; a camada de visualização (View), é a camada mais externa onde ocorrem as interações com o usuário, apresentando as páginas web e seus arquivos como o CSS, Javascript; e a camada de controle (Controller), recebe as interações do usuário com a camada de visualização e traduz em ações que devem ser desempenhadas com os métodos existentes no modelo, como pode ser exemplificado na Fig. 4.

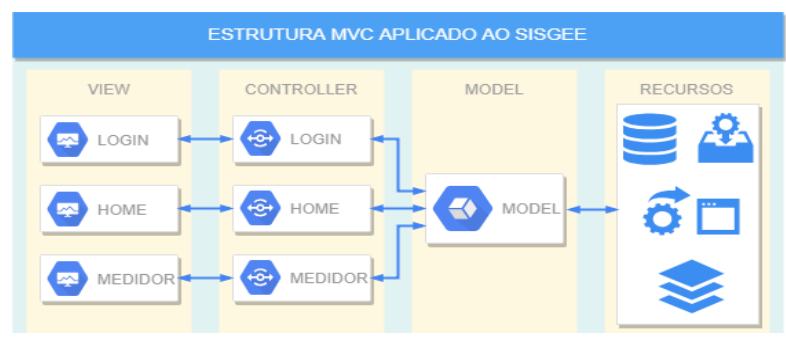

Fig. 4 - Estrutura do modelo MVC.

Como visto, a estrutura em camadas do MVC permite que ocorra a independência entre os componentes constituintes do sistema, de modo que o sistema alcance maior eficiência, escalabilidade, reutilização de métodos e classes, além da facilidade de manutenção.

\subsubsection{Material Design}

Os estudos provenientes na área de interface homem-máquina (IHM) levaram ao desenvolvimento do material design para a implementação de um sistema detentor de uma linguagem visual mais agradável e unificada. Dentre os princípios do material design, os conceitos de textura, tipografia, grades, movimento e o cruzamento de plataformas se sobressaem. O cruzamento de plataformas ocorre uma vez que, independente do sistema operacional que esteja em execução, os padrões de cores, traços e os demais modelos propostos pelo material design se sobreponham à diferença entre as plataformas, tornando o ambiente do software mais familiar.

A textura adotada com efeitos de sombra, contraste, tipografia e grades possuem a função de trazer a experiência de imersão para o usuário. Os movimentos e efeitos de transição possuem efeitos de continuidade e coerência de acordo com as dinâmicas encontradas no mundo físico. A Fig. 5 permite entender a pluralidade de acesso que acontecem a sistemas Web, em que o material design permite esta padronização de acesso.

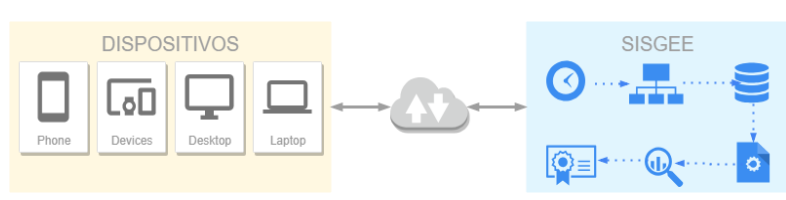

Fig. 5 - Acesso multiplataforma via WEB.

\subsubsection{Responsividade}

O modo de acessar informações por meio da internet também ficou muito mais abrangente, principalmente quando o assunto é tamanho de tela, pois existem diversos modelos de aparelhos telefônicos com diversas dimensões de tela, televisores e até mesmos relógios com acesso à internet. Sendo assim, por meio do design responsivo, foram criadas tecnologias para suportar a diversa gama de dispositivos, permitindo que o sistema web possa interpretar qual a melhor forma de exibir determinado conteúdo, além de considerar as características físicas e dimensionais dos equipamentos, proporcionando uma experiência agradável ao usuário, além de agregar valor ao produto final.

No caso do software SISGEE, as telas do sistema podem se adaptar ao dispositivo ao qual o usuário está fazendo acesso, como pode ser visto nas Fig. 6 e 7, que representam a tela de medidores do SISGEE e os conteúdos da navegação, respectivamente, tanto no modo Desktop/Notebook (I) quanto no modo Mobile (II). Desta forma, o software oferece a função de auto ajuste do sistema, alterando as fontes das letras, tamanho dos itens que constituem evitando que o usuário precise usar o zoom para verificar o conteúdo, ou seja, o software se adapta ao dispositivo e proporciona a comodidade ao usuário, redesenhando a tela em detrimento ao dispositivo que está sendo utilizado.

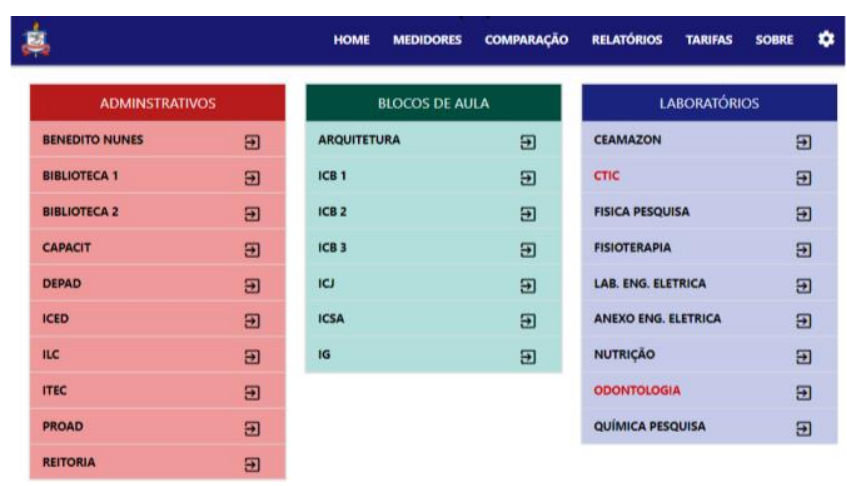

( I )

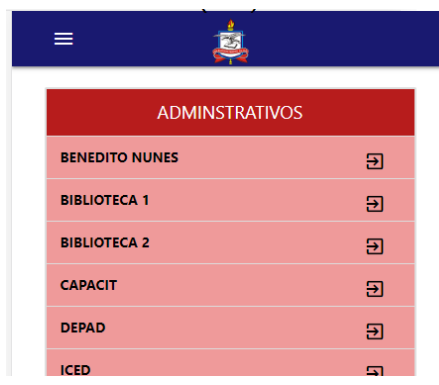

( II )

Fig. 6 - Tela de medidores: desktop (I) e mobile (II). 
क्ष

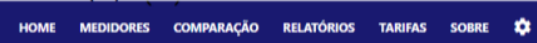
( I )
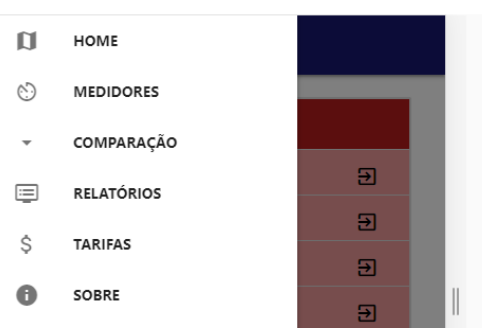

( II )

Fig. 7 - Disposição do menu: desktop (I) e mobile (II).

\section{APLICAÇÃO DO SOFTWARE E VALIDAÇÃO DE DADOS}

O software SISGEE possibilita a exportação dos dados medidos pelo sistema e, sendo assim, utilizou-se deste recurso para validar os dados medidos pelo medidor da EMBRASUL (sistema SISGEE), por meio da comparação dos dados com outro analisador de energia acoplado no mesmo ponto de medição. $\mathrm{O}$ analisador de energia portátil utilizado como referência foi o aparelho do fabricante HIOKI, modelo PW3198, que, além de calibrado, possui maior precisão. As medições foram realizadas no período de 19 a 25 de março de 2019, no PAC entre a UFPA e a Concessionária local.

Ambos medidores possibilitam a medição de diversas grandezas elétricas, contudo, este trabalho apresenta somente a comparação entre as grandezas de tensão e de corrente, que são as bases para o cálculo das demais grandezas elétricas do software.

\subsection{Aplicação do software}

Dentre as aplicações de análises do SISGEE, por meio da Fig. 8, é possível visualizar que os níveis de tensão medidos atendem os limites inferior e crítico do módulo 8 do PRODIST, para o nível de tensão de $13,8 \mathrm{kV}$. Contudo, no período analisado, percebe-se que a unidade consumidora (UC) obteve três quedas de tensão, ocasionando interrupção no funcionamento da mesma.

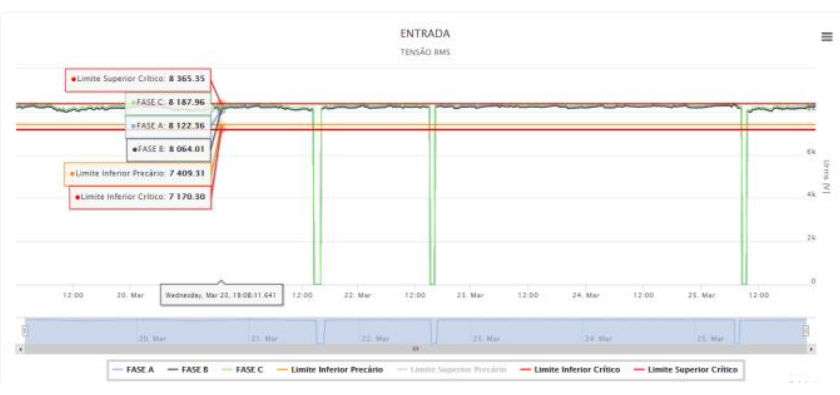

Fig. 8 - Análise de tensão no PAC da UC.

A Fig. 9 apresenta as medições de corrente no PAC, o que permite visualizar, tanto o perfil de carga da UC, quanto a saída das cargas durante o período analisado. O perfil de carga permite, também, visualizar os dias e horários em que a UC possui maior consumo, logo, esta informação possibilita os gestores a tomar decisões e efetuar ações de manutenção e eficiência energética.

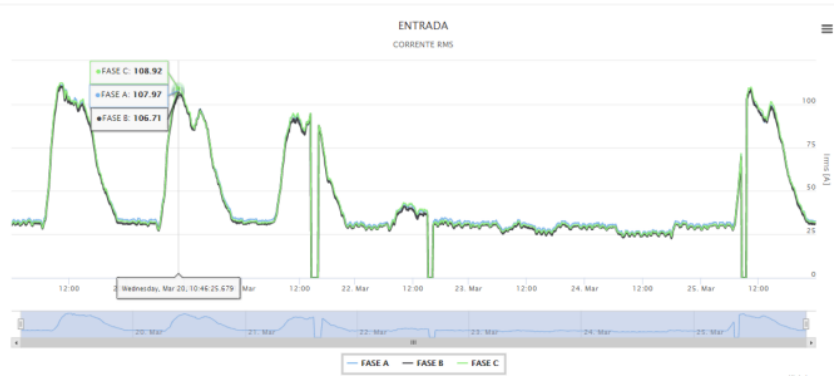

Fig. 9 - Análise de corrente no PAC da UC.

\subsection{Validação dos dados}

A Fig. 10 apresenta a comparação gráfica de um dia dos dados de tensão entre o sistema SISGEE (cor azul) e o analisador Hioki (cor vermelha), onde se pode visualizar a aproximação entre ambos.

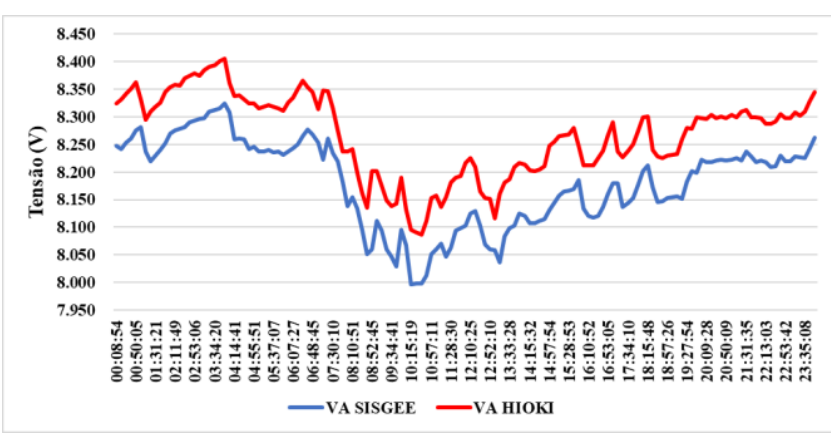

Fig. 10 - Gráficos de tensão do SISGEE versus Hioki.

Já a Tabela 1, mostra a comparação no período de uma semana entre os dois sistemas de medição. Em média quase $100 \%$ dos dados possui erro menor do que $2 \%$. E as fases A e $\mathrm{C}$ possuem erro menor do que $1 \%$, apresentando melhor precisão. Desta forma, para o período analisado, é possível verificar uma diferença mínima entre as tensões medidas.

Tabela 1 - Erro entre a tensão do SISGEE versus Hioki.

\begin{tabular}{|c|c|c|c|}
\hline Faixa & $V_{\mathbf{A}}(\boldsymbol{\%})$ & $\mathbf{V}_{\mathbf{B}}(\boldsymbol{\%})$ & $\mathrm{V}_{\mathbf{C}}(\boldsymbol{\%})$ \\
\hline $\mathbf{2 \%}<$ Erro $\leq \mathbf{3 \%}$ & 0,0 & 2,1 & 0,0 \\
\hline $\mathbf{1 \%}<$ Erro $\leq \mathbf{2 \%}$ & 3,1 & 96,3 & 1,2 \\
\hline Erro $\leq \mathbf{1 \%}$ & 96,9 & 1,6 & 98,8 \\
\hline
\end{tabular}

A comparação entre as correntes do sistema SISGEE (cor azul) e do analisador Hioki (cor vermelha) estão apresentadas na Fig. 11. Esta também permite destacar o perfil de carga da Universidade, em que, em um dia de semana, possui níveis de carregamento baixo de $00 \mathrm{~h}$ às $7 \mathrm{~h}$ da manhã e de $21 \mathrm{~h}$ às $23 \mathrm{~h} 59$. Independente do nível do carregamento, destaca-se a similaridade entre as correntes de ambos sistemas.

A Tabela 2, apresenta a diferença entre as correntes no período de uma semana entre os dois sistemas de medição. Nota-se que 
$95 \%$ do erro das três fases são menores do que 5\%. E uma média de $65,4 \%$ dos dados analisados possui incidência de medição na faixa de erro inferior a $1 \%$. A variação da corrente devido a oscilação da carga e o tempo de resposta dos analisadores, podem indicar os principais motivos para o maior erro entre os dados de corrente, deste estudo de caso.

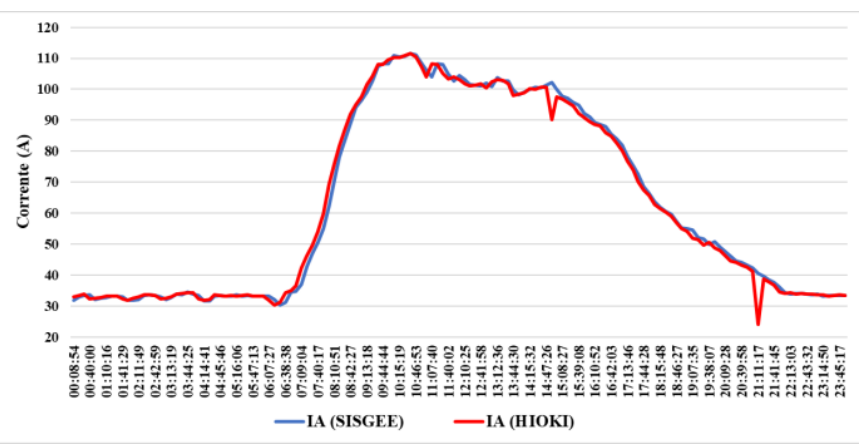

Fig. 11 - Erro entre a corrente do SISGEE versus Hioki.

Tabela 2 - Gráficos de corrente do SISGEE versus Hioki.

\begin{tabular}{|c|c|c|c|}
\hline Faixa & $\mathbf{I}_{\mathbf{A}}(\boldsymbol{\%})$ & $\mathbf{I}_{\mathbf{B}}(\boldsymbol{\%})$ & $\mathbf{I C}_{\mathbf{C}}(\boldsymbol{\%})$ \\
\hline Erro $>\mathbf{5 \%}$ & $4,7 \%$ & $5,7 \%$ & $5,1 \%$ \\
\hline $\mathbf{1 \%}<$ Erro $\leq \mathbf{5 \%}$ & $27,8 \%$ & $30,5 \%$ & $30,1 \%$ \\
\hline Erro $\leq \mathbf{1 \%}$ & $67,5 \%$ & $63,8 \%$ & $64,8 \%$ \\
\hline
\end{tabular}

5. CONCLUSÃO

Os valores encontrados na comparação de tensão e de corrente, entre os dados do SISGEE e do analisador HIOKI, apresentaram alto grau de confiabilidade. A análise da tensão mostrou que em 2 das 3 fases (A e C) medidas, o erro médio é menor do que $1 \%$, enquanto a fase $\mathrm{B}$ apresentou incidência de $96 \%$ de erro entre $1 \%$ e $2 \%$. Os dados de comparação da corrente entre os diferentes medidores, também foram satisfatórios para o monitoramento do campus universitário, haja vista que em média, $5 \%$ dos dados obteve erro maior do que $5 \%$, enquanto $65 \%$ obteve erro menor do que $1 \%$.

Além de se mostrar confiável, o sistema de gestão de energia apresentado permite melhor gerenciamento dos dados, otimização do tempo para tomada de ação e, consequentemente, redução de custos oriundos, se tornando ainda mais atrativo quando instalado em unidades consumidoras que possuem grandes dimensões de redes elétricas, além de baixo número de operadores e manutentores como é o caso do campus da UFPA.

\section{AGRADECIMENTOS}

Os Autores agradecem o apoio da CAPES, pelo financiamento 001, e do CNPq para a realização do presente trabalho, no âmbito do projeto "Sistema inteligente para determinação dos impactos harmônicos de múltiplos consumidores nas redes de distribuição de energia elétrica", aprovado na Chamada Universal - MCTI/CNPq/CT-ENERG No 14/2016.

\section{REFERÊNCIAS}

[1] G. L. Coutinho. A Era dos Smartphones: Um estudo exploratório sobre o uso dos smartphones no Brasil. 2015.

[2] S. Gulabani, Developing RESTful Web Services with Jersey 2.0, vol. I, 2015.

[3] C. Fortes, J. Santos, M. Reis, P. Poseiro, R. Capitão, L. Pinheiro, J. Craveiro, A. Sabino, A. Rodrigues, J. Ferreira, P. Raposeiro, S. Silva, E. Lopes, C. Silva, A. Simões, E. Azevedo e M. Rodrigues. HIDRALERTA - sistema de previsão e alerta de inundações em zonas costeiras e portuárias. DSpace, DHA/NPE - Comunicações a congressos e artigos de revista.2014.

[4] V. G. de Lima, F. de M. R. Magalhães, A. de O. Tito, R. A. dos Santos, A. R. R. Ristar, L. M. dos Santos, V. Vieira, A. C. Salgado. Ubibus Route: Um Sistema de Identificação e Sugestão de Rotas de Ônibus Baseado em Informações de Redes Sociais. Universidade Federal de Pernambuco. Recife, PE. 2012.

[5] B. M. Rahal, "Desenvolvimento de um Sistema de Monitoração e Validação para áreas de Transferência e Estocagem de uma Refinaria de Petróleo”. 2016.

[6] G. Bogueira, F. Batista, J. P. Carvalho. Sistema inteligente de recolha, armazenamento e visualização de informação proveniente do twitter. Atas da Conferência da Associação Portuguesa de Sistemas de Informação. Vol. 15. 2016.

[7] G. Stak. Módulo de I/O Remoto MODBUS. Universidade Federal do Rio Grande do Sul, Porto Alegre, RS. 2011.

[8] A. C. Krötz, Ferramenta e método para obtenção de parâmetros de confiabilidade fim-a-fim de redes industriais sem fio. 2019.

[9] MODBUS Application Protocol Specification. Acessível em <http://www.modbus.org/docs/Modbus_Application_ Protocol_V1_1b3.pdf $>$. Acessado em março de 2019.

[10] H. M. Carvalho, Aprendizado de Máquina voltado para Mineração de Dados: Árvores de Decisão. Universidade de Brasília, Brasília, DF. 2014.

[11] S. Eduardo, "Análise Comparativa na Utilização de Armaduras Soldadas e Armaduras Amarradas em Concreto Armado. Graduação em Engenharia Civil, 2019.

[12] Procedimentos de Distribuição de Energia Elétrica no Sistema Elétrico Nacional - PRODIST Módulo 8 Qualidade da Energia Elétrica. Acessível em: http://www2.aneel.gov.br/arquivos/pdf/modulo8_ revisao_1_retificacao_1.pdf >. Acessado em 22/03/2019.

[13] A. R. Duarte. Metodologia Rails: Análise da Arquitetura Model View Controller Aplicada. Universidade Federal de Minas Gerais, Minas Gerais, MG. 2011.

[15] Z. Tárcio. Web Design Responsivo: páginas adaptáveis para todos os dispositivos. Editora Casa do Código, 2015. 